\title{
ANÁLISE CONFORMACIONAL DE MODELOS DE LIGNINA ${ }^{\dagger}$
}

Hélio F. Dos Santos*

Departamento de Química, ICE, Universidade Federal de Juiz de Fora, Campus Universitário, Martelos, 36036-330, Juiz de Fora - MG

Recebido em 13/4/00; aceito em 10/11/00

CONFORMATIONAL ANALYSIS OF LIGNIN MODELS. The conformational equilibrium for two 5,5' biphenyl lignin models have been analyzed using a quantum mechanical semiempirical method. The gas phase and solution structures are discussed based on the NMR and X-ray experimental data. The results obtained showed that the observed conformations are solvent-dependent, being the geometries and the thermodynamic properties correlated with the experimental information. This study shows how a systematic theoretical conformational analysis can help to understand chemical processes at a molecular level.

Keywords: lignin models; conformational analysis; solvent effect; semiempirical methods.

\section{INTRODUÇÃO}

O termo lignina foi introduzido em 1838 por Anselme Payen $^{1}$ para designar o resíduo solúvel obtido no tratamento da madeira por ácido nítrico concentrado. Durante um longo período, a constituição química deste resíduo permaneceu obscura. Em 1917, Peter Klason ${ }^{1}$ propôs que a lignina poderia ser classificada como uma substância macromolecular constituída de unidades do álcool coniferílico mantidas juntas através de ligações do tipo éter. Em 1940, estudos baseados em reações clássicas da química orgânica, levaram a concluir que, de uma forma geral, a lignina era constituída de unidades fenilpropanóides unidas por ligações éter e carbono-carbono ${ }^{1}$ (Figura 1).

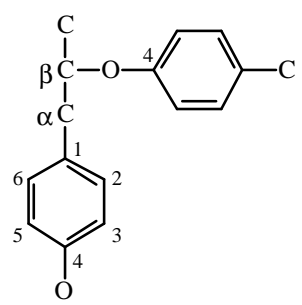

$\beta-\mathrm{O}-4$

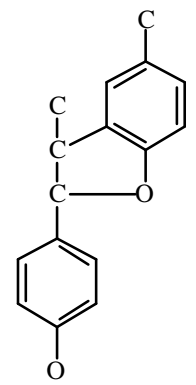

$\beta-5$

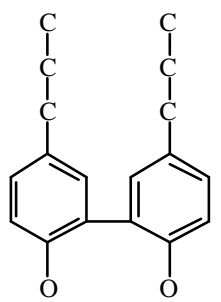

$5-5^{\prime}$
Figura 1. Principais tipos de ligações entre as unidades fenilpropanóides presentes na molécula de lignina.

Apesar das evidências experimentais, alguns cientistas contestavam a natureza aromática da lignina no seu estado nativo. Somente em 1954, Lange ${ }^{1}$ mostrou que os espectros no ultravioleta (UV) de seções finas da madeira eram característicos de compostos aromáticos e, de acordo com os estudos químicos desenvolvidos na década de 40, as principais unidades aromáticas presentes na estrutura da lignina foram classificadas como $p$-hidroxifenila, guaiacila e siringila (Figura 2).

\footnotetext{
* NEQC: Núcleo de Estudos em Química Computacional e-mail: helius@quimica.ufjf.br

† Este trabalho é dedicado a memória da Profa. Dra. Márcia A. Ferreira (DQ/UFJF)
}<smiles>Cc1ccc(O)cc1</smiles>

p-hidroxifenila<smiles>COc1cc(C)ccc1O</smiles>

guaiacila<smiles>COc1cc(C)cc(OC)c1O</smiles>

siringila
Figura 2. Principais unidades aromáticas presentes na molécula de lignina.

Baseado em informações obtidas através da análise dos tipos de ligações e grupos funcionais presentes, modelos estruturais para a lignina foram construídos. A primeira fórmula química para o sistema macromolecular foi proposta por Freudenberg ${ }^{1}$ em 1968 e confirmada posteriormente por outros pesquisadores ${ }^{1}$. Atualmente, o paradigma aceito para a estrutura da lignina é que a macromolécula está presente na madeira na forma de uma rede polimérica tridimensional não cristalina. Entretanto, alguns pesquisadores ${ }^{2}$ têm sugerido uma definição menos geral, baseada nas possíveis mudanças estruturais existentes em ligninas presentes em diferentes regiões morfológicas da madeira.

Nas últimas duas décadas, técnicas modernas de ressonância magnética nuclear (principalmente $\mathrm{RMN}$ de ${ }^{13} \mathrm{C}$ ) têm sido aplicadas na identificação e caracterização de subestruturas presentes na molécula de lignina ${ }^{3-5}$. Nestes estudos, pequenos fragmentos representativos do sistema macromolecular têm sido utilizados como compostos modelos. De acordo com a análise química dos resíduos obtidos em processos de polpeamento, as principais ligações presentes na lignina foram classificadas como $\beta-O-4(50 \%), \beta-5(9-12 \%)$ e $5,5^{\prime}(10-11 \%)^{1}$ (Figura 1). Portanto, a maioria dos trabalhos envolvendo compostos modelos utiliza moléculas contendo ligações desta natureza, sendo as espécies $5,5^{3,4}$ e $\beta-O-4^{5}$ as mais estudadas. Dentro do contexto da modelagem molecular, alguns trabalhos têm sido desenvolvidos utilizando modelos representantes da molécula de lignina ${ }^{5-8}$. Uma revisão da aplicação de métodos computacionais à química de ligninas pode ser encontrada na referência ${ }^{2}$.

No presente trabalho, a superfície de energia potencial (SEP) para os compostos 4,4'-O-dimetildesidrodiacetovanilona (DAM) e 4,4'-O-dietildesidrodiacetovanilona (DAE) foi analisada visando a determinação das principais estruturas localizadas em mínimos de energia na SEP e parâmetros relevantes para a análise conformacional. Estas moléculas foram sintetizadas recentemen$\mathrm{te}^{3} \mathrm{e}$ analisadas através de técnicas de $\mathrm{RMN}$ de ${ }^{1} \mathrm{H}$ e ${ }^{13} \mathrm{C}$. As estruturas cristalográficas para ambas as espécies foram 
obtidas $^{9}$, revelando aspectos estruturais interessantes que justificam o desenvolvimento de estudos teóricos.

\section{METOdOLOGIA DE CÁlCULO}

A análise conformacional teórica pode ser realizada utilizando metodologias sistemáticas ${ }^{10}$ e aleatórias ${ }^{11}$. No primeiro conjunto de métodos, a SEP é analisada através da variação de ângulos torcionais utilizando incrementos regulares ${ }^{10}$. A busca conformacional é baseada na análise combinatória de todos os possíveis ângulos de torção da molécula. Como consequiência, o número de estruturas geradas $(\mathrm{N})$ neste procedimento é significativamente elevado, sendo função do incremento utilizado (A) e do número de ligações consideradas nos processos de rotação $(\mathrm{T})$ (eq. 1).

$$
N=\left(\frac{360}{A}\right)^{T}
$$

$\mathrm{Na}$ análise conformacional aleatória ${ }^{11}$, a SEP é investigada através de movimentos aleatórios nas posições dos átomos. As estruturas geradas são aceitas ou não de acordo com janelas energéticas definidas pelo analista em diferentes etapas do algoritmo de busca conformacional ${ }^{11}$.

A aplicabilidade de metodologias sistemáticas ou aleatórias é definida em função do sistema a ser analisado. De uma forma geral, a análise conformacional aleatória é recomendada para moléculas contendo anéis saturados, susceptíveis a torções intramoleculares ${ }^{12,13}$. Para moléculas que apresentam rotações internas, a análise conformacional sistemática é mais apropriada ${ }^{14-16}$. Neste contexto, quando o interesse do analista se limita na localização das principais conformações da molécula, um caminho alternativo pode ser utilizado, no qual a SEP é mapeada através da construção de projeções tridimensionais (3D-SEP) geradas pela rotação simultânea de diferentes pares de ângulos diedros. As conformações de interesse (mínimos e máximos na SEP) são obtidas diretamente da inspeção das superfícies e mapas de contorno. Este procedimento tem sido aplicado com sucesso em alguns estudos anteriores ${ }^{14-18}$ e foi utilizado no presente trabalho para a análise conformacional das moléculas DAM e DAE.

O esquema de numeração e definição dos principais ângulos diedros envolvidos nos processos de interconversão conformacional para as moléculas DAM e DAE são apresentados na Figura 3. Devido à alta flexibilidade torcional dos sistemas analisados, um procedimento de busca conformacional sistemática foi estabelecido e aplicado na investigação das SEP, visando à identificação das principais conformações localizadas em mínimos de energia. Nesta estratégia de análise conformacional, diferentes representações tridimensionais da SEP (3D-SEP) foram construídas utilizando pares distintos dos ângulos diedros definidos na Figura 3.

Em uma primeira etapa, a 3D-SEP envolvendo o ângulo interanéis $\left(\omega_{1} \times \omega_{2}\right)$ foi construída e as conformações estáveis obtidas agrupadas em dois conjuntos (syn e anti) de acordo com a definição topológica apresentada no Esquema 1.

A estrutura de menor energia em cada um dos conjuntos foi utilizada como ponto de partida na construção da superfície $\omega_{2} \times \omega_{3}$. O mesmo procedimento foi seguido na construção das 3D-SEP $\omega_{2} \times \omega_{4}, \omega_{3} \times \omega_{5}$ e $\omega_{6} \times \omega_{7}$. Todas as superfícies foram construídas considerando os comprimentos e ângulos de ligação fixos em valores previamente otimizados. Um total de 9 representações tridimensionais da SEP foram construídas para cada molécula, sendo calculados 324 pontos em cada uma das superfícies. O Esquema 2 apresenta a seqüência de etapas envolvida no procedimento de análise conformacional utilizado neste estudo.

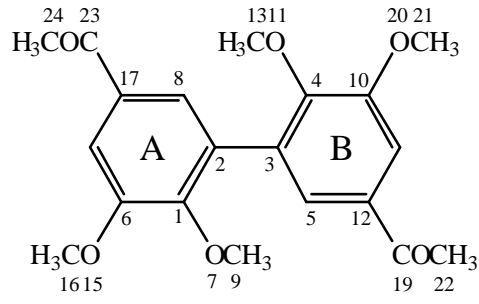

(a)

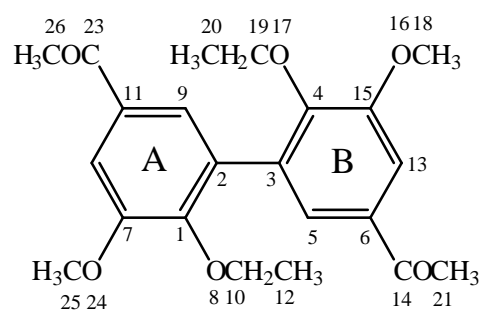

(b)

Figura 3. Esquema de numeração e definição dos principais ângulos diedros das espécies diméricas DAM (3a) $\left(w_{1}:\left[C_{4}, C_{3}, C_{2}, C_{1}\right] w_{2}:\left[C_{9}, O_{7}, C_{1}, C_{2}\right]\right.$ $w_{3}:\left[C_{13}, O_{11}, C_{4}, C_{3}\right] w_{4}:\left[C_{16}, O_{15}, C_{6}, C_{1}\right] w_{5}:\left[C_{21}, O_{20}, C_{10}, C_{4}\right] w_{6}:\left[C_{22}, C_{19}, C_{12}, C_{5}\right]$ $\left.w_{7}:\left[C_{24}, C_{23}, C_{17}, C_{8}\right]\right)$ e DAE (3b) $\left(w_{1}:\left[C_{4}, C_{3}, C_{2}, C_{1}\right] w_{2}:\left[C_{10}, O_{8}, C_{1}, C_{2}\right]\right.$ $w_{3}:\left[C_{19}, O_{17}, C_{4}, C_{3}\right] w_{4}:\left[C_{25}, O_{24}, C_{7}, C_{1}\right] w_{5}:\left[C_{18}, O_{16}, C_{15}, C_{13}\right] w_{6}:\left[C_{21}, C_{14}, C_{6}, C_{5}\right]$ $\left.w_{7}:\left[C_{26}, C_{23}, C_{11}, C_{9}\right] ; w_{8}:\left[C_{12}, C_{10}, O_{8}, C_{1}\right] w_{9}:\left[C_{20}, C_{19}, O_{17}, C_{4}\right]\right)$.

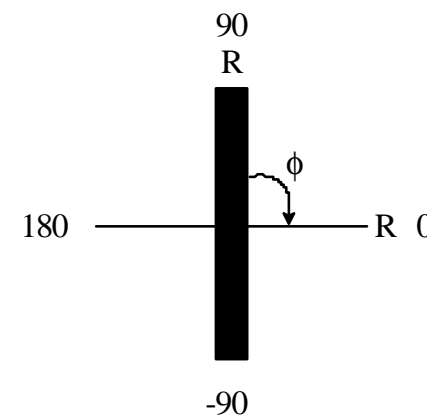

syn: $\{\phi \in \mathfrak{X}(0<\phi<90) \cap(-90<\phi<0)\} ;$ anti: $\{\phi \in \mathfrak{X}(90<\phi<180) \cap(180<\phi<-90)\}$

Esquema 1. Definição do espaço conformacional syn $e$ anti utilizado neste trabalho.

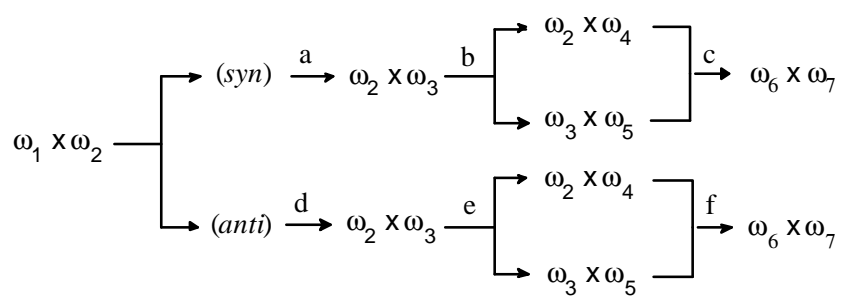

Esquema 2. Procedimento utilizado na análise conformacional dos compostos diméricos DAM e DAE (Figura 3). Os ângulos torcionais $w_{i}$ são definidos na Figura 3 e a denominação syn $e$ anti é apresentada no Esquema 1.

Todas as conformações obtidas em pontos de mínimos na SEP foram completamente otimizadas e caracterizadas como mínimos verdadeiros através do cálculo dos autovalores da matriz Hessiana (todos os autovalores positivos caracterizam um ponto estacionário como um mínimo de energia ${ }^{19}$ ). Os 
estados de transição envolvidos nos processos de interconversão conformacional syn $\rightarrow$ anti foram otimizados sem restrição e caracterizados através do cálculo das freqüências vibracionais ${ }^{19}$. Todos os cálculos foram realizados utilizando a aproximação semi-empírica AM1 (Austin Model 1) ${ }^{20}$ implementada no programa MOPAC versão $7.0^{21}$. O seguinte conjunto de keywords foi utilizado no processo de otimização das geometrias: $\mathrm{EF}^{22}$ (TS para os estados de transição), PRE$\mathrm{CISE}^{21}$ e GNORM=0.1 $1^{21}$.

$\mathrm{O}$ efeito do solvente foi incluído utilizando o modelo contínuo COSMO (Conductor-like Screening Model) ${ }^{23}$ com os valores dos parâmetros NSPA (número de segmentos por átomo) $=60 \mathrm{e} \mathrm{R}^{\text {solv }}=\delta^{\text {SC }}=1,0 \AA^{23}$. A contribuição estrutural para o processo de solvatação foi analisada através da otimização das estruturas na presença do meio dielétrico (solvente).

\section{RESULTADOS E DISCUSSÃO}

\section{Análise conformacional na fase gasosa}

As Tabelas 1 e 2 apresentam os valores otimizados dos principais ângulos diedros (Figura 3) obtidos para as distintas conformações localizadas em mínimos de energia na SEP para as moléculas DAM e DAE respectivamente. As diferentes estruturas foram classificadas e agrupadas como syn (simbolizadas por $\mathrm{s}_{\mathrm{i}}$ ) e anti (simbolizadas por $\mathrm{a}_{\mathrm{i}}$ ) de acordo com a definição topológica apresentada no Esquema 1. Na segunda coluna das Tabelas 1 e 2 são representados os pares dos ângulos torcionais utilizados na construção das diferentes representações 3D-SEP (Esquema 2). Como pode ser visto, conformações estruturalmente distintas foram obtidas em diferentes superfícies, mostrando a necessidade de se fazer um mapeamento sistemático e exaustivo da hipersuperfície de energia potencial.

Analisando as estruturas apresentadas na Tabela 1 (molécula DAM), pode ser visto que as conformações $a_{1}-a_{3}$ e $s_{1}-s_{3}$ se diferenciam, principalmente, pela posição relativa dos grupos $\mathrm{OCH}_{3}$ ligados em $\mathrm{C}_{1}$ e $\mathrm{C}_{4}$ (Figura 3a) caracterizada pelos ângulos torcionais $\omega_{2}$ e $\omega_{3}$. As geometrias $a_{4}-a_{7}$ e $s_{4}-s_{6}$ apresentam torções diferenciadas nos grupamentos $\mathrm{O}=\mathrm{C}\left(\mathrm{CH}_{3}\right)$ (ver valores de $\omega_{6}$ e $\omega_{7}$, Tabela 1$)$. Com relação aos substituintes $\mathrm{OCH}_{3}$ nas posições $\mathrm{C}_{6}$ e $\mathrm{C}_{10}$, pequenas variações foram observadas, sendo os valores de $\omega_{4}$ e $\omega_{5}$ próximos de 180 graus. Para a molécula DAE (Tabela 2), um maior número de conformações foi obtido em função da maior flexibilidade conformacional dos substituintes $\mathrm{OCH}_{2} \mathrm{CH}_{3}$ ligados nas posições $\mathrm{C}_{1}$ e $\mathrm{C}_{4}$ dos anéis $\mathrm{A}$ e $\mathrm{B}$ respectivamente (Figura $3 \mathrm{~b}$ ). As conformações $a_{7}, a_{12}, s_{3}, s_{5}$ e $s_{6}$ são caracterizadas pelos valores de $\omega_{6}$ e $\omega_{7}$, os quais especificam torções nos grupamentos $\mathrm{O}=\mathrm{C}\left(\mathrm{CH}_{3}\right)$. É importante observar que as mudanças conformacionais no conjunto de estruturas classificadas como syn são restritas, em consequiência das repulsões estéricas entre os substituintes ligados nas posições orto dos anéis $\mathrm{A}$ e $\mathrm{B}$.

$\mathrm{Na}$ Tabela 3 são relatados os valores relativos de energia total $\left(\Delta \mathrm{E}^{\text {gás }}\right)$ e energia livre de Gibbs $\left(\Delta \mathrm{G}^{\text {gás }}\right)$ calculados para as distintas conformações apresentadas nas Tabelas 1 e 2 . Os resultados mostram que para ambos os sistemas, a conformação anti (a $(\mathrm{DAM})$ e $\mathrm{a}_{2}$ (DAE)) foi obtida como mínimo global na SEP, sendo a diferença de energia livre de Gibbs $\left(\Delta G^{\text {gás }}\right)$ entre as conformações syn e anti igual a 0,64 (DAM, $\mathrm{s}_{3}-\mathrm{a}_{1}$ ) e $1,74 \mathrm{kcal} / \mathrm{mol}$ (DAE, $\mathrm{s}_{1}-\mathrm{a}_{2}$ ). É importante mencionar que apesar da conformação $a_{1}$ (DAE) possuir menor energia que $a_{2}$, a inclusão da correção térmica $(H-$ TS) altera a estabilidade conformacional. De acordo com os valores de $\Delta \mathrm{G}^{\text {gás }}$ apresentados na Tabela 3, as energias relativas entre as diferentes formas possuem valores menores que $4 \mathrm{kcal} / \mathrm{mol}$. Este resultado pode ser relacionado ao procedimento de busca conformacional utilizado (Esquema 2), o qual visa a obtenção das principais (mais prováveis) estruturas presentes em equilíbrio.

A maior estabilidade da conformação anti pode ser entendida considerando aspectos qualitativos relacionados à repulsão estérica e conjugação eletrônica. Neste contexto, os valores do ângulo torcional inter-anéis $\omega_{1}$ (Figura 3 e Tabelas 1 e 2) podem ser utilizados para avaliar as intensidades destes efeitos nas conformações syn e anti. Para as estruturas classificadas como anti, os valores absolutos de $\omega_{1}$ foram obtidos próximos de $140^{\circ}$, enquanto as formas syn apresentaram valores próximos de $80^{\circ}$. Este resultado demonstra que o desvio da planaridade $\left(\omega_{1}=180^{\circ}\right.$ para anti e $\omega_{1}=0^{\circ}$ para syn) é menor para as conformações anti e consequentemente a conjugação eletrônica entre os anéis aromáticos contribui de forma mais significativa para a estabilidade conformacional. Estes mesmos argumentos foram aplicados para justificar a ordem de estabilidade

Tabela 1. Conformações distintas localizadas em mínimos de energia na hipersuperfície de energia potencial para a molécula DAM, segundo o procedimento de análise conformacional aplicado neste estudo. Os principais ângulos diedros são representados por $\omega_{\mathrm{i}}$ (em graus). Os resultados foram obtidos considerando o sistema isolado (fase gasosa).

\begin{tabular}{|c|c|c|c|c|c|c|c|c|}
\hline \multicolumn{9}{|c|}{ Molécula DAM } \\
\hline & \multirow[b]{2}{*}{ 3D-SEP ${ }^{c}$} & \multicolumn{7}{|c|}{ Principais ângulos diedros ${ }^{\mathrm{a}, \mathrm{b}}$} \\
\hline & & $\omega_{1}$ & $\omega_{2}$ & $\omega_{3}$ & $\omega_{4}$ & $\omega_{5}$ & $\omega_{6}$ & $\omega_{7}$ \\
\hline$a_{1}$ & $\omega_{1} \times \omega_{2}$ & -135 & 117 & 142 & -176 & -179 & 2 & 2 \\
\hline$a_{2}$ & $\omega_{1} \times \omega_{2}$ & 134 & -141 & -141 & 180 & 180 & -2 & -1 \\
\hline$a_{3}$ & $\omega_{3} \times \omega_{5}$ & -135 & 118 & 118 & -176 & -175 & 2 & 4 \\
\hline$a_{4}$ & $\omega_{6} \times \omega_{7}$ & -134 & 116 & 148 & -172 & -177 & 4 & -163 \\
\hline$a_{5}$ & $\omega_{6} \times \omega_{7}$ & -134 & 119 & 140 & -174 & -175 & -163 & 6 \\
\hline$a_{6}$ & $\omega_{3} \times \omega_{5}$ & -135 & 119 & 72 & -175 & 51 & 12 & 7 \\
\hline$a_{7}$ & $\omega_{6} \times \omega_{7}$ & -134 & 117 & 146 & -168 & -168 & -167 & -168 \\
\hline $\mathrm{s}_{1}$ & $\omega_{3} \times \omega_{5}$ & 80 & -112 & 146 & 174 & -177 & 9 & 1 \\
\hline $\mathrm{s}_{2}$ & $\omega_{2} \times \omega_{3}$ & 80 & -114 & -151 & 174 & 180 & 6 & 6 \\
\hline $\mathrm{s}_{3}$ & $\omega_{1} \times \omega_{2}$ & 78 & -113 & 119 & 175 & -175 & 10 & -2 \\
\hline $\mathrm{s}_{4}$ & $\omega_{6} \times \omega_{7}$ & 79 & -112 & 143 & 171 & -179 & 3 & 170 \\
\hline $\mathrm{s}_{5}$ & $\omega_{6} \times \omega_{7}$ & 76 & -115 & 143 & 174 & -175 & 163 & -7 \\
\hline $\mathrm{s}_{6}$ & $\omega_{6} \times \omega_{7}$ & 78 & -112 & 141 & 170 & -178 & 168 & -166 \\
\hline
\end{tabular}

${ }^{\mathrm{a}}$ Ver Figura 3a para definição dos ângulos diedros $\omega_{\mathrm{i}} ;{ }^{\mathrm{b}} \mathrm{O}$ sinal negativo refere-se a definição do ângulo torcional no sentido antihorário e positivo no sentido horário; ${ }^{\mathrm{c}} \omega_{\mathrm{i}}$ e $\omega_{\mathrm{j}}$ representam os ângulos diedros utilizados na construção da superfície de energia potencial tridimensional (Figura 3a). 
Tabela 2. Conformações distintas localizadas em mínimos de energia na hipersuperfície de energia potencial para a molécula DAE, segundo o procedimento de análise conformacional aplicado neste estudo. Os principais ângulos diedros são representados por $\omega_{\mathrm{i}}$ (em graus). Os resultados foram obtidos considerando o sistema isolado (fase gasosa).

\begin{tabular}{|c|c|c|c|c|c|c|c|c|c|c|}
\hline \multicolumn{11}{|c|}{ Molécula DAM } \\
\hline & \multirow[b]{2}{*}{$3 \mathrm{D}-\mathrm{SEP}^{\mathrm{c}}$} & \multicolumn{9}{|c|}{ Principais ângulos diedros ${ }^{\mathrm{a}, \mathrm{b}}$} \\
\hline & & $\omega_{1}$ & $\omega_{2}$ & $\omega_{3}$ & $\omega_{4}$ & $\omega_{5}$ & $\omega_{6}$ & $\omega_{7}$ & $\omega_{8}$ & $\omega_{9}$ \\
\hline$a_{1}$ & $\omega_{1} \times \omega_{2}$ & 136 & -139 & -139 & 176 & -4 & 4 & 4 & 150 & 150 \\
\hline$a_{2}{ }^{d}$ & $\omega_{3} \times \omega_{5}$ & 136 & -115 & -140 & 173 & -5 & 9 & 7 & -179 & 150 \\
\hline$a_{3}{ }^{d}$ & $\omega_{3} \times \omega_{5}$ & 136 & -140 & -114 & 176 & -112 & 15 & 8 & 150 & -176 \\
\hline$a_{4}$ & $\omega_{2} \times \omega_{3}$ & 132 & 154 & -138 & -176 & 0 & -8 & 5 & -158 & 149 \\
\hline$a_{5}$ & $\omega_{1} \times \omega_{2}$ & -135 & 114 & -150 & -173 & -5 & -10 & -18 & 179 & 152 \\
\hline$a_{6}{ }^{d}$ & $\omega_{3} \times \omega_{5}$ & 136 & -116 & -130 & 174 & -69 & 15 & -1 & -180 & -124 \\
\hline$a_{7}$ & $\omega_{6} \times \omega_{7}$ & 134 & -143 & -138 & 174 & -8 & 165 & -1 & 152 & 148 \\
\hline$a_{8}$ & $\omega_{2} \times \omega_{3}$ & 134 & -138 & 131 & 179 & 8 & 8 & 2 & 149 & 125 \\
\hline$a_{9}$ & $\omega_{1} \times \omega_{2}$ & -129 & -157 & -157 & 169 & -12 & 3 & 3 & 160 & 160 \\
\hline$a_{10}$ & $\omega_{3} \times \omega_{5}$ & 132 & -138 & 124 & 180 & 107 & -4 & -6 & 149 & -172 \\
\hline$a_{11}$ & $\omega_{3} \times \omega_{5}$ & 136 & -141 & -72 & 174 & 133 & -12 & 6 & 150 & 179 \\
\hline$a_{12}$ & $\omega_{6} \times \omega_{7}$ & 134 & -142 & -142 & 165 & -16 & 170 & 170 & 151 & 151 \\
\hline$a_{13}$ & $\omega_{2} \times \omega_{3}$ & 133 & 152 & 132 & -170 & 9 & 18 & 3 & -153 & 124 \\
\hline $\mathrm{s}_{1}$ & $\omega_{1} \times \omega_{2}$ & 81 & -111 & 143 & 174 & 4 & 9 & -1 & -180 & -152 \\
\hline $\mathrm{s}_{2}$ & $\omega_{2} \times \omega_{3}$ & 80 & 119 & -111 & -174 & -7 & -2 & 11 & -175 & -179 \\
\hline $\mathrm{s}_{3}$ & $\omega_{6} \times \omega_{7}$ & 80 & -111 & 141 & 170 & 3 & 3 & 172 & -180 & -151 \\
\hline $\mathrm{s}_{4}$ & $\omega_{3} \times \omega_{5}$ & 79 & -112 & 115 & 174 & 112 & 9 & -3 & 180 & 177 \\
\hline $\mathrm{S}_{5}$ & $\omega_{6} \times \omega_{7}$ & 77 & -114 & 140 & 173 & 8 & 163 & -7 & -179 & -151 \\
\hline $\mathrm{s}_{6}$ & $\omega_{6} \times \omega_{7}$ & 79 & -111 & 139 & 170 & 5 & 170 & -165 & -180 & -150 \\
\hline
\end{tabular}

${ }^{\mathrm{a}}$ Ver Figura 3b para definição dos ângulos diedros $\omega_{i}$; ${ }^{\mathrm{b}} \mathrm{O}$ sinal negativo refere-se a definição do ângulo torcional no sentido antihorário e positivo no sentido horário; ${ }^{\mathrm{c}} \omega_{\mathrm{i}}$ e $\omega_{\mathrm{j}}$ representam os ângulos diedros utilizados na construção da superfície de energia potencial tridimensional (Figura 3b); ${ }^{\mathrm{d}}$ Estruturas obtidas após completa otimização da geometria localizada em ponto de mínimo na SEP para a conformação syn.

Tabela 3. Energia total relativa $\left(\Delta \mathrm{E}^{\text {gás }}\right)$ e energia livre de Gibbs relativa $\left(\Delta \mathrm{G}^{\text {gás }}, \mathrm{T}=298 \mathrm{~K}\right)$ para as distintas conformações das moléculas DAM e DAE calculadas na fase gasosa, utilizando o método AM1 (valores em $\mathrm{kcal} / \mathrm{mol}$ ). O momento de dipolo elétrico é também apresentado (valores em Debye).

\begin{tabular}{|c|c|c|c|c|c|c|}
\hline & \multicolumn{3}{|c|}{ DAM } & \multicolumn{3}{|c|}{ DAE } \\
\hline & $\Delta \mathrm{E}^{\mathrm{gás}}$ & $\Delta \mathrm{G}^{\text {gás }}$ & $\mu$ & $\Delta \mathrm{E}^{\mathrm{gás}}$ & $\Delta \mathrm{G}^{\text {gás }}$ & $\mu$ \\
\hline $\mathrm{a}_{1}$ & 0,00 & 0,00 & 2,106 & 0,00 & 0,00 & 2,592 \\
\hline$a_{2}$ & 0,01 & 0,05 & 2,071 & 0,14 & $-0,28$ & 2,849 \\
\hline$a_{3}$ & 0,02 & 0,16 & 1,998 & 0,63 & 0,43 & 2,974 \\
\hline$a_{4}$ & 1,22 & 1,23 & 4,798 & 0,77 & 0,36 & 1,288 \\
\hline$a_{5}$ & 1,48 & 1,39 & 5,536 & 0,92 & 1,25 & 2,317 \\
\hline$a_{6}$ & 2,61 & 1,72 & 4,019 & 1,23 & 0,66 & 1,865 \\
\hline$a_{7}$ & 2,64 & 2,57 & 4,181 & 1,35 & 1,44 & 5,293 \\
\hline$a_{8}$ & & & & 1,60 & 1,41 & 1,120 \\
\hline $\mathrm{a}_{9}$ & & & & 1,73 & 1,20 & 0,164 \\
\hline$a_{10}$ & & & & 1,73 & 1,02 & 2,393 \\
\hline$a_{11}$ & & & & 2,47 & 2,07 & 4,643 \\
\hline$a_{12}$ & & & & 2,61 & 3,05 & 3,981 \\
\hline$a_{13}$ & & & & 2,69 & 2,74 & 0,541 \\
\hline $\mathrm{s}_{1}$ & 1,48 & 0,78 & 2,123 & 1,61 & 1,46 & 2,138 \\
\hline $\mathrm{s}_{2}$ & 1,55 & 1,10 & 2,359 & 1,86 & 1,57 & 1,733 \\
\hline $\mathrm{s}_{3}$ & 1,57 & 0,64 & 1,613 & 2,06 & 1,91 & 4,501 \\
\hline $\mathrm{s}_{4}$ & 1,92 & 1,23 & 4,434 & 2,39 & 1,83 & 2,622 \\
\hline $\mathrm{S}_{5}$ & 2,27 & 1,54 & 5,612 & 2,42 & 1,67 & 5,654 \\
\hline $\mathrm{s}_{6}$ & 2,91 & 2,12 & 4,047 & 3,04 & 2,83 & 4,175 \\
\hline
\end{tabular}

entre as diferentes formas isoméricas obtidas para os produtos de reações de ciclotrimerização de cetonas acetilênicas substituídas ${ }^{16}$. Em um estudo anterior desenvolvido por Remko e colaboradores ${ }^{6}$, a SEP para o composto 2,2'dimetoxibifenila apresentou um único mínimo localizado em $\omega_{1}=135^{\circ}$. Este resultado está em perfeito acordo com os va- lores determinados para as conformações anti das moléculas DAM e DAE (Tabelas 1 e 2). De acordo com os valores dos ângulos inter-anéis obtidos para as conformações da espécie DAE (Tabela 2), pode ser observado que a inclusão de um grupamento metileno ao substituinte alcóxi ligado às posições orto dos anéis aromáticos não provoca alterações signi- 


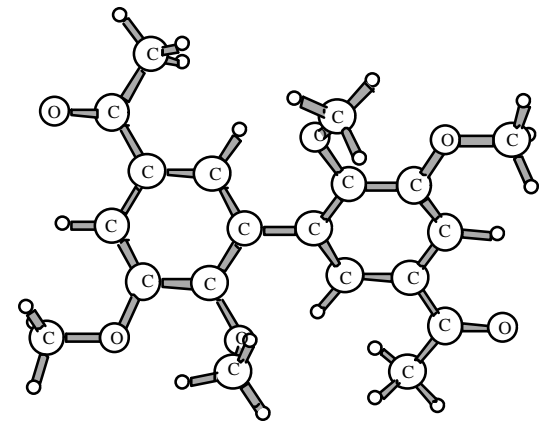

DAM-anti $\left(\mathrm{a}_{1}\right) ; \Delta \mathrm{G}^{\text {gás }}=0,00 \mathrm{kcal} / \mathrm{mol}$

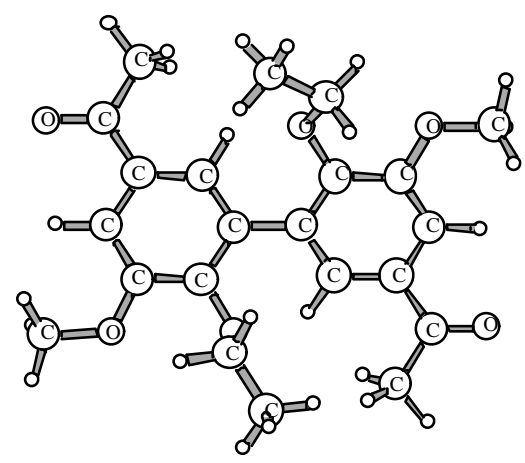

$\operatorname{DAE}-\operatorname{anti}\left(\mathrm{a}_{2}\right) ; \Delta \mathrm{G}^{\text {gás }}=0,00 \mathrm{kcal} / \mathrm{mol}$

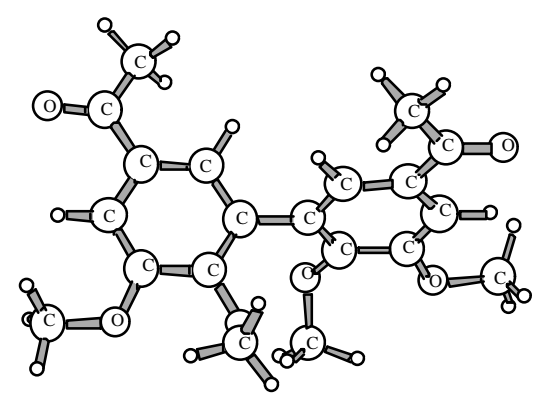

DAM-syn $\left(\mathrm{s}_{3}\right) ; \Delta \mathrm{G}^{\text {gás }}=0,64 \mathrm{kcal} / \mathrm{mol}$

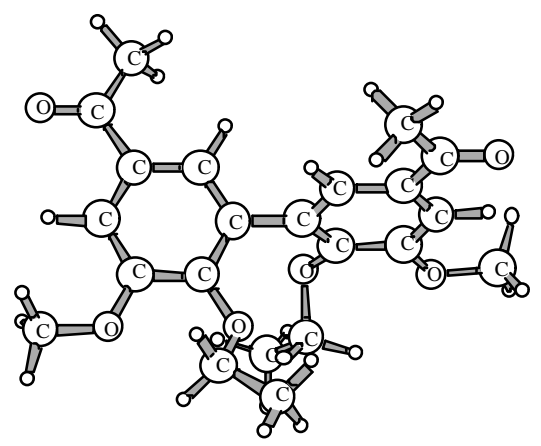

DAE- $\operatorname{syn}\left(\mathrm{s}_{1}\right) ; \Delta \mathrm{G}^{\text {gás }}=1,74 \mathrm{kcal} / \mathrm{mol}$

Figura 4. Estruturas otimizadas (AMI) para as conformações anti e syn de menor energia das moléculas DAM e DAE.

ficativas nas posições relativas dos anéis benzênicos. As representações das estruturas tridimensionais para as conformações otimizadas $a_{1}$ e $s_{3}$ (DAM) e $a_{2}$ e $s_{1}$ (DAE) são apresentadas na Figura 4.

As estruturas cristalográficas dos compostos DAM e DAE foram determinadas através de técnicas de difração de raios$\mathrm{X}^{9}$. A conformação obtida para a molécula DAM no estado sólido apresentou uma estrutura simétrica com um ângulo inter-anéis $\left(\omega_{1}\right)$ igual a $60^{\circ}$, sendo portanto, caracterizada como syn, de acordo com a definição apresentada no Esquema 1. A conformação de menor energia para o composto DAM classificada como syn (estrutura $s_{3}$, Tabelas 1 e 3 ), apresentou um valor para o ângulo torcional $\omega_{1}$ igual a $78^{\circ}$. Em uma primeira análise, as discrepâncias entre teoria e experimento podem ser justificadas em termos das interações intermoleculares presentes na rede cristalina (todos os cálculos foram realizados considerando os sistemas como um gás ideal). Trabalhos experimentais relacionados à molécula de bifenila, mostraram um valor para o ângulo inter-anéis igual a $44^{\circ}$ na fase gasosa ${ }^{24}$ e $0^{\circ}$ (conformação plana) no estado sólido $^{25}$, evidenciando a importância das interações intermoleculares no estado sólido. Os demais ângulos diedros, definidos na Figura 3a, apresentaram os seguintes valores para a conformação observada no estado sólido para o composto DAM (em graus): $\omega_{2}=\omega_{3}=-96, \omega_{4}=\omega_{5}=179$ e $\omega_{6}=\omega_{7}$ $=10$. Os valores obtidos para a conformação $\mathrm{s}_{3}$ foram (em graus): $-113,119,175,-175,10$ e -2 respectivamente para os ângulos diedros $\omega_{2}-\omega_{7}$ (Tabela 1). Analisando os valores apresentados anteriormente, pode ser visto que as principais diferenças estruturais são relacionadas às posições espaciais relativas dos grupos metóxi $\left(\mathrm{OCH}_{3}\right)$ ligados nas posições $\mathrm{C}_{1}$ e $\mathrm{C}_{4}$, as quais são definidas pelo par de ângulos torcionais $\omega_{2}$ e $\omega_{3}$. De acordo com os dados cristalográficos, os átomos de oxigênio pertencentes aos substituintes $\mathrm{OCH}_{3}$ ligados às posições orto dos anéis aromáticos, estão separados por $2,94 \AA$, gerando uma estrutura energeticamente desfavorável devido à repulsão eletrostática entre centros carregados negativamente.
A conformação obtida pelo método AM1 apresentou uma distância $\mathrm{O} \ldots \mathrm{O}$ igual a $3,41 \AA$, sendo à distância $\mathrm{H}\left(\mathrm{CH}_{3}\right) \ldots \mathrm{O}$ igual a 2,40 $\AA$, favorecendo uma interação eletrostática atrativa entre os átomos de $\mathrm{H}\left(\mathrm{CH}_{3}\right)$ e $\mathrm{O}$.

A Figura 5 representa o mapa de contorno para uma região da 3D-SEP $\omega_{2} \times \omega_{3}$ correspondente a conformação syn da molécula DAM. As estruturas estáveis $s_{2}$ e $s_{3}$ (Tabela 1) são indicadas com o objetivo de demonstrar o baixo valor para a barreira de energia rotacional envolvida no processo $\mathrm{s}_{3} \rightarrow \mathrm{s}_{2}$ $\left(\Delta \mathrm{E}^{\#} \approx 3 \mathrm{kcal} / \mathrm{mol}\right)$. Esta interconversão conformacional é relacionada à rotação de um grupo $\mathrm{OCH}_{3}$ (definido por $\omega_{3}$ ) de 90 graus, justificando a possibilidade de existência da estrutura observada no estado sólido em função das interações intermoleculares presentes na rede cristalina.

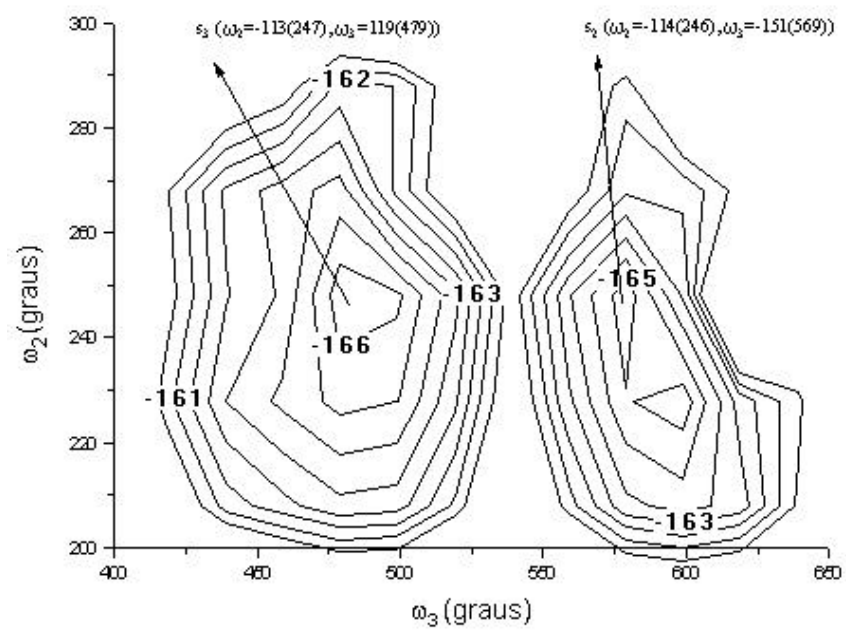

Figura 5. Mapa de contorno de uma região da $3 D-P E S w_{2} x w_{3}$ para a conformação syn da molécula DAM. As estruturas $s_{2}$ e $s_{3}$ estão localizadas em pontos de energia mínima na superfície. 
Os dados cristalográficos relativos à molécula $\mathrm{DAE}^{9}$, revelaram a presença de duas conformações distintas no estado sólido, sendo uma das formas classificada como anti $\left(\omega_{1}=115^{\circ}\right)$ e a outra como syn $\left(\omega_{1}=60^{\circ}\right)$. Os valores apresentados na Tabela 2 mostram que as estruturas de menor energia pertencentes aos conjuntos anti e syn (estruturas $\mathrm{a}_{2}$ e $\mathrm{s}_{1}$ ) possuem um ângulo inter-anéis igual a $136\left(\mathrm{a}_{2}\right)$ e $81^{\circ}\left(\mathrm{s}_{1}\right)$ respectivamente. Devido à presença de duas estruturas distintas na unidade assimétrica, é esperado que as discrepâncias entre teoria e experimento sejam mais pronunciadas, como conseqüência da não consideração das interações intermoleculares no tratamento teórico. Os valores dos demais ângulos torcionais $\left(\omega_{2}-\omega_{9}\right)$ para as conformações presentes no estado sólido são (em graus) (valores entre parênteses correspondem aos respectivos ângulos diedros calculados para as conformações $\mathrm{a}_{2}$ e $\left.\mathrm{s}_{1}\right)$ : anti: $96(-115),-112(-$ 140), -172(173), -10(-5), -5(9), 7(7), -153(-179) e 170(150); syn: -111(-111), 128(143), -179(174), -11(4), 177(9), 15(-1), 151(-180) e -154(-152). A conformação syn, presente no estado sólido, apresenta uma torção no ângulo $\omega_{6}$ de 168 graus em relação à estrutura $s_{1}$. Esta mudança conformacional pode ser justificada em termos do baixo valor da barreira rotacional para este processo. A Figura 6 mostra a representação da 3D-SEP $\omega_{6} \times \omega_{7}$ para a conformação syn da molécula DAE. A barreira de energia envolvida no processo $\mathrm{s}_{1} \rightarrow \mathrm{s}_{5}$ é de apenas $2,1 \mathrm{kcal} /$ mol, sendo a conformação $s_{5}\left(\omega_{6}=163^{\circ}\right)$ equivalente à estrutura cristalográfica com relação à posição relativa dos grupamentos $\mathrm{O}=\mathrm{C}\left(\mathrm{CH}_{3}\right)$.

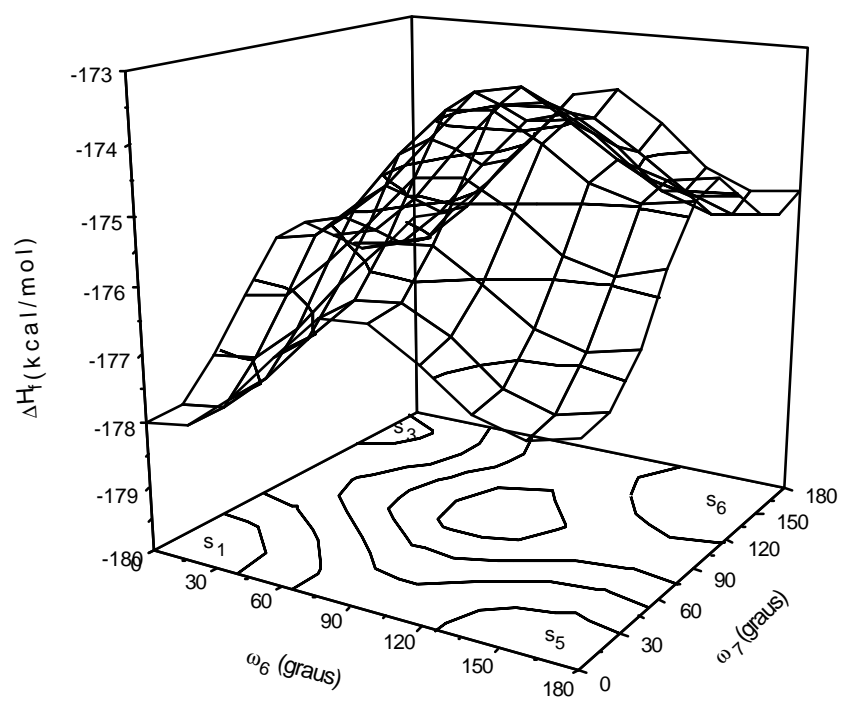

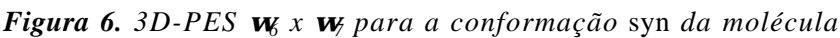
$D A E$. As estruturas localizadas em mínimos de energia são indicadas por $s_{1}, s_{3}, s_{5} e s_{6}$ (ver Tabela 2$)$.

Com o objetivo de verificar se as estruturas cristalográficas representam pontos estacionários na $\mathrm{SEP}$, as geometrias obtidas no estado sólido foram utilizadas como pontos iniciais no procedimento de minimização de energia. Os resultados obtidos nesta análise para a molécula DAM, levaram a uma estrutura equivalente a forma $\mathrm{a}_{2}$ apresentada na Tabela 1 , a qual corresponde a uma geometria de equilíbrio localizada apenas $0,05 \mathrm{kcal} / \mathrm{mol}$ acima do mínimo global $\left(\mathrm{a}_{1}\right)$.

Para o derivado DAE, as conformações anti e syn, observadas no estado sólido, foram otimizadas separadamente. As conformações obtidas após o procedimento de minimização de energia são correspondentes as formas $a_{4}$ e $s_{5}$ (ver Tabelas 2 e 3 ).

A última etapa do procedimento de análise conformacional na fase gasosa, envolveu o estudo das barreiras de energia relacionadas aos processos de interconversão entre as formas syn e anti. Três estruturas correspondentes aos estados de transição
(ET) envolvidos nas interconversões anti $\rightarrow$ syn, syn $\rightarrow$ syn $\mathrm{e}$ anti $\rightarrow$ anti foram completamente otimizadas e caracterizadas como pontos de máximo na SEP através do cálculo das frequiências vibracionais. Os principais parâmetros estruturais e os valores obtidos para as barreiras de energia são apresentados na Tabela 4. Os resultados mostraram uma pequena barreira de energia para a interconversão anti $\rightarrow$ syn, a qual nos leva a concluir que este processo deve ocorrer com velocidade considerável em temperaturas relativamente baixas. Considerando a pequena diferença de energia entre as conformações syn e anti (ver Tabela 3), pode ser dito que o equilíbrio entre as duas formas apresenta características favoráveis do ponto de vista cinético e termodinâmico na fase gasosa.

Devido aos aspectos particulares relacionados a estereoquímica de bifenilas ${ }^{26}$, uma determinada parte da literatura afim tem sido dedicada ao estudo do atropisomerismo em moléculas desta natureza ${ }^{26-30}$. A possibilidade de isomeria ótica em compostos bifenílicos, é conhecida desde 1922 quando Christie e Kenner conseguiram resolver em duas formas ativas o ácido 2,2'-dinitrodifenil-6,6'-dicarboxílico. A atividade ótica em bifenilas é determinada por fatores relacionados à restrição da rotação ao redor da ligação inter-anéis. Estudos envolvendo processos de racemização destas moléculas ${ }^{27-30}$ têm mostrado que o valor para a energia de ativação necessária para que o composto apresente isomeria ótica deve ser > $20 \mathrm{kcal} / \mathrm{mol}$. É importante mencionar que a racemização nestas moléculas ocorre via um estado de transição no qual ambos os anéis aromáticos encontram-se no mesmo plano, portanto, de acordo com a natureza dos substituintes ligados nas posições orto dos anéis benzênicos, as barreiras rotacionais podem assumir valores significativos.

No presente estudo, as barreiras energéticas envolvidas nos processos de racemização das moléculas DAM e DAE foram calculadas utilizando o método AM1. Para estes sistemas em particular, a racemização pode ocorrer via dois caminhos. No primeiro destes processos (envolvendo a estrutura ET2), as barreiras energéticas calculadas apresentaram valores de 15,77 (DAM) e 17,93 kcal/mol (DAE). A interconversão envolvendo o estado de transição ET3 apresentou os seguintes valores para a energia de ativação: 9,68 (DAM) e 9,42 kcal/mol (DAE). Portanto, de acordo com os resultados obtidos, podemos concluir que não seria observado atividade ótica nestes compostos devido aos baixos valores para a energia de racemização.

\section{Análise conformacional em solução}

Como mencionado na introdução deste trabalho, a maioria dos estudos envolvendo modelos de lignina, utiliza técnicas de RMN na identificação estrutural e correlação com dados relativos ao sistema macromolecular. Considerando moléculas simples, técnicas de RMN podem ser úteis na determinação de aspectos relacionados às conformações presentes no meio (alguns exemplos podem ser encontrados nas referências ${ }^{31,32}$ ). Entretanto, informações desta natureza são de difícil acesso quando moléculas complexas são analisadas. Neste contexto, considerando que algumas técnicas espectroscópicas (RMN, UV, etc.) são aplicadas a soluções, a inclusão do efeito do solvente no processo de análise conformacional teórica pode auxiliar o experimentalista no tratamento de dados fornecidos pela espectroscopia.

No presente trabalho, o modelo contínuo $\mathrm{COSMO}^{23}$ foi utilizado para a inclusão do efeito do solvente na análise conformacional das moléculas DAM e DAE. As diferentes estruturas obtidas na fase gasosa e apresentadas nas Tabelas 1 e 2 foram completamente otimizadas na presença dos solventes clorofórmio $\left(\mathrm{CHCl}_{3} ; \varepsilon=4,806\right)$ e dimetilsulfóxido (DMSO, $\left.\varepsilon=45,0\right)$. Devido à baixa solubilidade dos sistemas estudados, o número de solventes de interesse foi limitado. Os resultados obtidos mostraram uma pequena variação dos parâmetros geométricos devido às interações do soluto com o meio. As diferenças estruturais entre as geometrias obtidas na fase gasosa e em solução 
Tabela 4. Propriedades relacionadas aos diferentes estados de transição (ET1-ET3) envolvidos nos processos de interconversão conformacional das moléculas DAM e DAE.

\begin{tabular}{|c|c|c|c|c|c|c|c|c|c|c|c|c|}
\hline \multicolumn{13}{|c|}{ Ângulos diedros otimizados ${ }^{\mathrm{a}, \mathrm{b}}$ (graus) } \\
\hline Estrutura & a Processo & $\omega_{1}$ & $\omega_{2}$ & $\omega_{3}$ & $\omega_{4}$ & $\omega_{5}$ & $\omega_{6}$ & $\omega_{7}$ & $\omega_{8}$ & $\omega_{9}$ & $\begin{array}{c}\Delta \mathrm{H}_{\mathrm{f}} \\
(\mathrm{kcal} / \mathrm{mol})\end{array}$ & $\begin{array}{c}\Delta \mathrm{E}^{\# \mathrm{c}} \\
(\mathrm{kcal} / \mathrm{mol})\end{array}$ \\
\hline \multicolumn{13}{|c|}{ Molécula DAM $^{\mathrm{d}}$} \\
\hline ET1 & anti $\rightarrow$ syn & -93 & 114 & 142 & -174 & -179 & -5 & -6 & & & $-166,69$ & 1,69 \\
\hline ET2 & syn $\rightarrow$ syn & -3 & -149 & 112 & -178 & -170 & -13 & -14 & & & $-151,04$ & 15,77 \\
\hline ET3 & anti $\rightarrow$ anti & -179 & -111 & -129 & 173 & 175 & -17 & -17 & & & $-158,70$ & 9,68 \\
\hline \multicolumn{13}{|c|}{ Molécula DAE } \\
\hline ET1 & anti $\rightarrow$ syn & -99 & 113 & -117 & -176 & -8 & -9 & 2 & -179 & 179 & $-177,88$ & 1,99 \\
\hline ET2 & syn $\rightarrow$ syn & -4 & -134 & 127 & 170 & 10 & -14 & -12 & -126 & 138 & $-160,46$ & 17,93 \\
\hline ET3 & anti $\rightarrow$ anti & 180 & 111 & -111 & -173 & -8 & -7 & 7 & 166 & -1660 & $-170,45$ & 9,42 \\
\hline
\end{tabular}

${ }^{\mathrm{a}}$ Os principais ângulos torcionais para as moléculas DAM e DAE são representados respectivamente nas Figuras $3 \mathrm{a}$ e $3 \mathrm{~b}$; ${ }^{\mathrm{b}} \mathrm{O}$ sinal negativo refere-se a definição do ângulo diedro no sentido anti-horário e positivo no sentido horário; ${ }^{\mathrm{c}} \Delta \mathrm{E}^{\#}$ corresponde a barreira de energia para a interconversão conformacional, definida como: $\mathrm{E}_{\mathrm{ET} 1}-\mathrm{E}_{\mathrm{a} 1}\left(\right.$ anti $\rightarrow$ syn), $\mathrm{E}_{\mathrm{ET} 2}-\mathrm{E}_{\mathrm{S} 3}(s y n \rightarrow s y n)$ e $\mathrm{E}_{\mathrm{ET} 3}-\mathrm{E}_{\mathrm{a} 1}$ $\left(\right.$ anti $\rightarrow$ anti) $(\mathrm{DAM}) ; \mathrm{E}_{\mathrm{ET} 1}-\mathrm{E}_{\mathrm{a} 2}\left(\right.$ anti $\rightarrow$ syn), $\mathrm{E}_{\mathrm{ET} 2}-\mathrm{E}_{\mathrm{S} 1}\left(s y n \rightarrow\right.$ syn) e $\mathrm{E}_{\mathrm{ET} 3}-\mathrm{E}_{\mathrm{a} 2}$ (anti $\rightarrow$ anti) (DAE); ${ }^{\mathrm{O}}$ cálculo de freqüências vibracionais para os estados de transição (ET) envolvidos nos processos de interconversão conformacional no composto DAM forneceu os seguintes valores imaginários $\left(\mathrm{em} \mathrm{cm}^{-1}\right)$ : 19,9i (ET1), 72,4i (ET2) e 64,5i (ET3); ${ }^{\mathrm{e} O}$ cálculo de freqüências vibracionais para os estados de transição (ET) envolvidos nos processos de interconversão conformacional no composto DAE forneceu os seguintes valores imaginários $\left(\mathrm{em} \mathrm{cm}^{-1}\right)$ : 14,4i (ET1), 53,3i (ET2) e 51,2i (ET3).

foram avaliadas através do cálculo do RMS (root mean square) utilizando as coordenadas espaciais de ambas as formas. Os valores máximos de RMS foram (em $\AA$ ): 0,122 (DAM, $\mathrm{CHCl}_{3}$ ); 0,366 (DAM, DMSO); 0,176 (DAE, $\mathrm{CHCl}_{3}$ ) e 0,300 (DAE, DMSO). No geral, as distorções estruturais na presença de DMSO foram mais pronunciadas. Devido às pequenas modificações geométricas causadas pelas interações soluto...solvente, a contribuição estrutural para o processo de solvatação é relativamente pequena.

A estabilidade relativa entre as diferentes conformações em solução foi calculada utilizando o ciclo termodinâmico apresentado no Esquema 3 , onde $\Delta \mathrm{G}_{\mathrm{ij}}{ }^{\text {gás }}$ corresponde aos valores de energia livre de Gibbs relativa apresentados na Tabela 3 (DAM, conf. $._{\mathrm{i}}=\mathrm{a}_{1}$; DAE, conf. ${ }_{\mathrm{i}}=\mathrm{a}_{2}$ ), $\Delta \mathrm{G}^{\text {solv }}$ representa a energia livre de solvatação das conformações i e j e $\Delta \mathrm{G}_{\mathrm{ij}}{ }^{\text {sol }}$ a variação de energia livre em solução calculada utilizando a eq. (2).

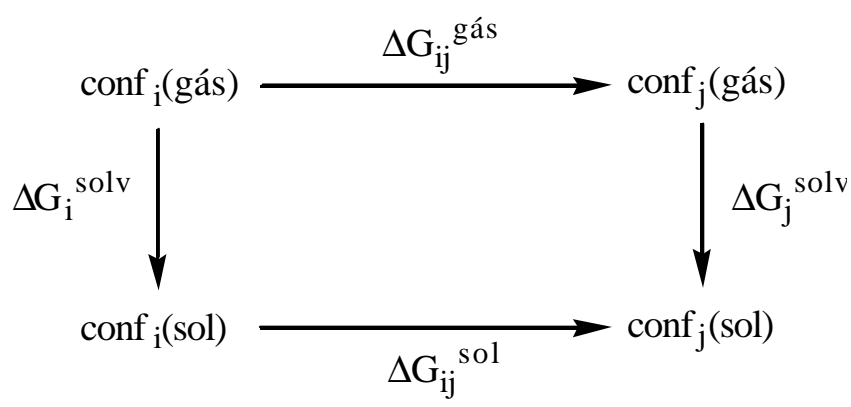

Esquema 3. Ciclo termodinâmico utilizado no cálculo da energia livre de Gibbs relativa em solução para as diferentes conformações das moléculas DAM e DAE.

$\Delta \mathrm{G}_{\mathrm{ij}}^{\text {sol }}=\Delta \mathrm{G}_{\mathrm{ij}}^{\text {gás }}+\left(\Delta \mathrm{G}_{\mathrm{j}}^{\text {solv }}-\Delta \mathrm{G}_{\mathrm{i}}^{\text {solv }}\right)$

Os resultados obtidos para as conformações mais estáveis são apresentados na Tabela 5. Os valores representados na Tabela 5 mostram que, de uma forma geral, o equilíbrio é deslocado para a forma syn com o aumento da constante dielétrica do meio. Para o composto DAM, a conformação syn $\left(s_{3}\right)$ foi obtida como mínimo global em $\mathrm{CHCl}_{3}$ e DMSO. Para o derivado DAE, a forma anti $\left(\mathrm{a}_{2}\right.$ em $\mathrm{CHCl}_{3}$ e $\mathrm{a}_{9}$ em DMSO) apresentou energia ligeiramente inferior a conformação syn $\left(\mathrm{s}_{2}\right.$ em $\mathrm{CHCl}_{3}$ e $\mathrm{s}_{5}$ em DMSO) em solução.
Tabela 5. Energia livre de Gibbs relativa entre as conformações mais estáveis classificadas como anti e syn na fase gasosa e em solução. Os valores de energia foram obtidos utilizando as geometrias otimizadas na fase gasosa e em solução.

\begin{tabular}{lcccc}
\hline & $\Delta \mathrm{G}^{\text {gás }}$ & \multicolumn{3}{c}{$\Delta \mathrm{G}^{\text {sol }}$} \\
\hline DAM & Gás & $\mathrm{CHCl}_{3}$ & Etanol & DMSO \\
\hline anti & $0,00\left(\mathrm{a}_{1}\right)$ & $0,80\left(\mathrm{a}_{3}\right)$ & $0,90\left(\mathrm{a}_{6}\right)$ & $0,91\left(\mathrm{a}_{7}\right)$ \\
syn & $0,64\left(\mathrm{~s}_{3}\right)$ & $0,00\left(\mathrm{~s}_{3}\right)$ & $0,00\left(\mathrm{~s}_{3}\right)$ & $0,00\left(\mathrm{~s}_{3}\right)$ \\
\hline DAE & Gás & $\mathrm{CHCl}_{3}$ & Etanol & DMSO \\
\hline anti & $0,00\left(\mathrm{a}_{2}\right)$ & $0,00\left(\mathrm{a}_{2}\right)$ & $0,00\left(\mathrm{a}_{9}\right)$ & $0,00\left(\mathrm{a}_{9}\right)$ \\
syn & $1,74\left(\mathrm{~s}_{1}\right)$ & $0,41\left(\mathrm{~s}_{2}\right)$ & $0,33\left(\mathrm{~s}_{5}\right)$ & $0,29\left(\mathrm{~s}_{5}\right)$ \\
\hline
\end{tabular}

Os cristais utilizados nas medidas de difração de raios-X para as moléculas DAM e DAE foram obtidos através de recristalização em etanol ${ }^{9}$. Considerando a hipótese de que a conformação observada no estado sólido corresponde àquela de menor energia presente em solução ${ }^{33}$ (etanol), cálculos foram realizados com a inclusão do efeito do solvente definido pela constante dielétrica macroscópica do etanol $(\varepsilon=24,30)$. Todas as geometrias apresentadas nas Tabelas 1 e 2 foram completamente otimizadas na presença do meio dielétrico e a estabilidade relativa calculada utilizando a eq. (2). Os resultados obtidos apresentaram características intermediárias aos estudos envolvendo $\mathrm{CHCl}_{3}$ e DMSO e são apresentados na Tabela 5.

Estes resultados podem ser correlacionados com as estruturas observadas no estado sólido. Considerando que somente as conformações apresentadas na Tabela 5 estão presentes no meio, os valores da variação de energia livre de Gibbs em etanol para o processo anti $\rightarrow$ syn levam a seguinte distribuição de população: DAM: $18 \%$ (anti) e $82 \%$ (syn); DAE: $64 \%$ (anti) e $36 \%$ (syn). Portanto, esta análise demonstra que o derivado DAM encontra-se predominantemente na forma syn em solução de etanol, justificando a presença de uma única conformação no estado sólido caracterizada como syn. Para a molécula DAE, a razão de população syn/anti foi igual a 0,56 , o que pode ser utilizado como evidência da presença de ambas as conformações na rede cristalina. Um outro aspecto importante desta análise é a presença da conformação $s_{5}$ para o derivado DAE em etanol, a qual é equivalente a estrutura cristalográfica caracterizada como syn.

Estudos de Ressonância Magnética Nuclear foram realiza- 
dos em $\mathrm{CDCl}_{3}\left(\mathrm{RMN}{ }^{1} \mathrm{H}\right)$ e DMSO-d $6\left(\mathrm{RMN}^{13} \mathrm{C}\right)^{3}$. Os resultados apresentaram sinais característicos de uma única espécie simétrica em solução, entretanto, aspectos estruturais não foram relatados. De acordo com os valores apresentados na Tabela 5, a molécula DAM encontra-se presente na conformação syn em solução, sendo a população relativa igual a: $79 \%$ $\left(\mathrm{CHCl}_{3}\right)$ e $82 \%$ (DMSO). O derivado DAE apresentou uma predominância da conformação anti, $67 \%\left(\mathrm{CHCl}_{3}\right)$ e $62 \%$ (DMSO), com a forma syn presente em quantidades consideráveis: $33 \%\left(\mathrm{CHCl}_{3}\right)$ e $38 \%$ (DMSO). As geometrias otimizadas em DMSO para as conformações $s_{3}$ (DAM) e $a_{9}$ e $s_{5}$ (DAE) são apresentadas na Figura 7.

Em uma última análise envolvendo o efeito do solvente nas propriedades relacionadas ao equilíbrio conformacional, as geometrias cristalográficas obtidas para as moléculas DAM e $\mathrm{DAE}^{9}$ foram submetidas ao processo de minimização de energia na presença do meio dielétrico. $\mathrm{O}$ objetivo deste estudo foi analisar o efeito das interações eletrostáticas soluto...solvente na estrutura e estabilidade das conformações correspondentes às formas presentes no estado sólido. Devido ao fato de que a molécula DAM apresentou uma única conformação no estado sólido, uma análise mais sistemática foi desenvolvida para este sistema. Como mencionado anteriormente, a utilização da estrutura cristalográfica da molécula DAM como ponto de partida no procedimento de minimização de energia na fase gasosa levou à conformação $\mathrm{a}_{2}$. Este mesmo procedimento foi utilizado considerando diferentes valores de constante dielétrica $(\varepsilon)$. Os resultados obtidos são apresentados na Tabela 6 .

Como pode ser visto na Tabela 6 , a integridade da confor- mação observada no estado sólido é mantida para valores de $\varepsilon>15$. Para valores de $\varepsilon<10$ a conformação obtida no processo de otimização de geometria é caracterizada como anti segundo os valores de $\omega_{1}$ (Esquema 1). Considerando as estruturas obtidas nessa análise, os valores de $\Delta \mathrm{E}$ em etanol e DMSO, calculados em relação à forma $\mathrm{s}_{3}$, foram: $-0,21$ e $-0,18$ $\mathrm{kcal} / \mathrm{mol}$ respectivamente, sendo a conformação $\mathrm{s}_{3}$ energeticamente desfavorecida. A geometria otimizada em DMSO $\left(\mathrm{c}_{14}\right.$, Tabela 6) é mostrada na Figura 8.

$\mathrm{Na}$ Figura 9 é representado a variação do ângulo interanéis $\left(\omega_{1}\right)$ em função da constante dielétrica do meio $(\varepsilon)$. Um comportamento assintótico foi observado, sendo o valor de $\omega_{1}$ invariante em solventes com constante dielétrica maior que 20. Este resultado pode ser justificado analisando as aproximações do modelo COSMO. A metodologia COSMO foi desenvolvida considerando a teoria de condutores ${ }^{23}$. Com o objetivo de descrever propriedades de sistemas moleculares em solventes com constante dielétrica finita, uma função empírica $(f(\varepsilon)$, eq. 3) foi introduzida na energia de interação soluto...solvente.

$$
f(\varepsilon)=\frac{2(\varepsilon-1)}{(2 \varepsilon+1)}
$$

De acordo com a eq. (3), $\mathrm{f}(\varepsilon) \rightarrow 1$ quando $\varepsilon \rightarrow \infty$, sendo convergente para $\varepsilon>20$. Esta análise pode ser utilizada como justificativa para o comportamento apresentado na Tabela 6 e Figura 9.

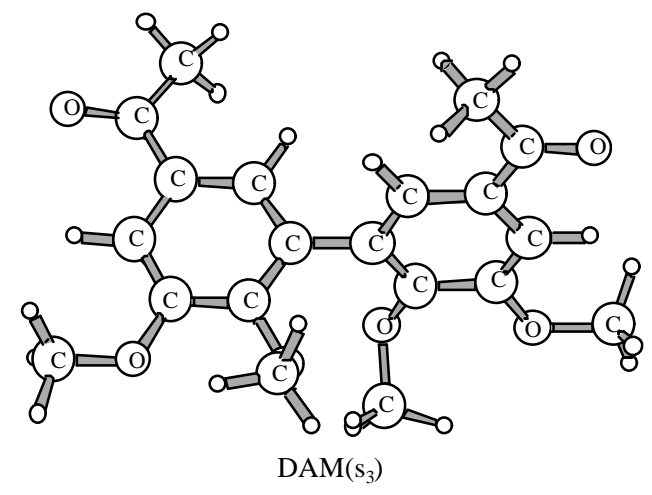

(a)

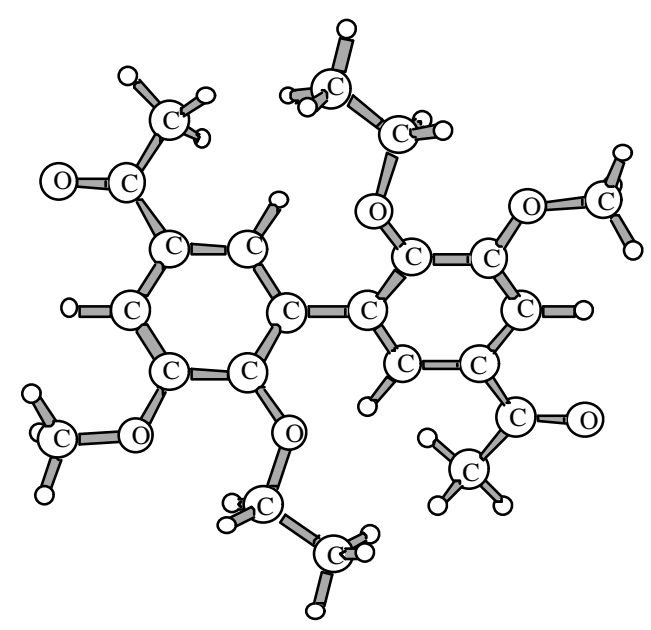

$\operatorname{DAE}\left(\mathrm{a}_{9}\right)$

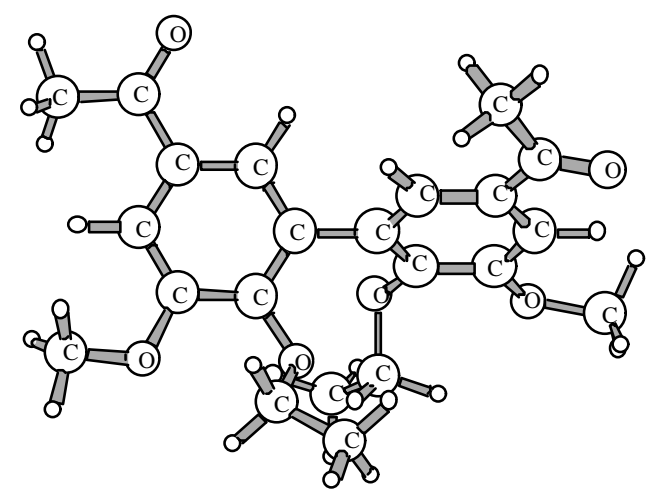

$\operatorname{DAE}\left(\mathrm{s}_{5}\right)$

(b)

Figura 7. Estruturas otimizadas em DMSO. (7a) DAM, $s_{3}$ e (7b) DAE, a $a_{9}$ e $s_{5}$. 
Tabela 6. Influência do solvente na estrutura da molécula DAM como função da constante dielétrica macroscópica ( $\varepsilon$ ). As geometrias otimizadas em diferentes solventes foram obtidas utilizando a estrutura cristalográfica como ponto de partida. Os ângulos diedros $\omega_{\mathrm{i}}$ são definidos na Figura $3 \mathrm{a}$.

\begin{tabular}{|c|c|c|c|c|c|c|c|c|c|}
\hline Solvente & $\varepsilon^{\mathrm{a}}$ & & $\omega_{1}$ & $\omega_{2}$ & $\omega_{3}$ & $\omega_{4}$ & $\omega_{5}$ & $\omega_{6}$ & $\omega_{7}$ \\
\hline Gás b & 1,0 & $c_{1}=a_{2}$ & -134 & 141 & 141 & 180 & 180 & 2 & 2 \\
\hline Cicloexano & 2,023 & $\mathrm{c}_{2}$ & -134 & -144 & -146 & 177 & 178 & -11 & -11 \\
\hline $\mathrm{CCl}_{4}$ & 2,238 & $\mathrm{c}_{3}$ & -126 & -123 & -123 & 176 & 177 & -10 & -10 \\
\hline Benzeno (Bz) & 2,284 & $\mathrm{c}_{4}$ & -126 & -123 & -123 & 177 & 177 & -11 & -11 \\
\hline $\mathrm{CHCl}_{3}$ & 4,806 & $\mathrm{c}_{5}$ & -115 & -117 & -117 & -178 & -178 & -14 & -14 \\
\hline$\left(\mathrm{CH}_{3}\right)_{2} \mathrm{O}$ & 7,9 & $\mathrm{c}_{6}$ & -116 & -117 & -117 & -174 & -174 & -15 & -15 \\
\hline o-dicloro-Bz & 9,93 & $\mathrm{c}_{7}$ & -115 & -117 & -116 & -172 & -172 & -16 & -16 \\
\hline 2-butanol & 15,8 & $\mathrm{c}_{8}$ & -89 & -116 & -116 & -170 & -170 & 17 & 17 \\
\hline 2-MeO-etanol & 16,9 & $\mathrm{c}_{9}$ & -88 & -116 & -116 & -170 & -170 & 17 & 17 \\
\hline 1-butanol & 17,8 & $\mathrm{c}_{10}$ & -87 & -116 & -116 & -170 & -170 & 18 & 18 \\
\hline Acetona & 20,7 & $\mathrm{c}_{11}$ & -88 & -116 & -116 & -169 & -169 & 18 & 18 \\
\hline $\mathrm{EtOH}$ & 24,30 & $c_{12}$ & -87 & -116 & -116 & -169 & -169 & 18 & 18 \\
\hline $\mathrm{CH}_{3} \mathrm{OH}$ & 32,63 & $\mathrm{c}_{13}$ & -83 & -117 & -117 & -167 & -167 & 19 & 19 \\
\hline DMSO & 45,0 & $\mathrm{c}_{14}$ & -82 & -116 & -116 & -167 & -167 & 19 & 19 \\
\hline $\mathrm{H}_{2} \mathrm{O}$ & 78,54 & $c_{15}$ & -81 & -117 & -117 & -166 & -166 & 19 & 19 \\
\hline Sólido ${ }^{c}$ & & & -60 & -96 & -96 & 179 & 179 & 10 & 10 \\
\hline
\end{tabular}

${ }^{\mathrm{a}}$ Constante dielétrica obtida da referência [34]; ${ }^{\mathrm{b}}$ Geometria correspondente à estrutura $\mathrm{a}_{2}$ (Tabela 1); ${ }^{\mathrm{c}}$ Estrutura obtida por difração de raios-X (ver ref. [9]).

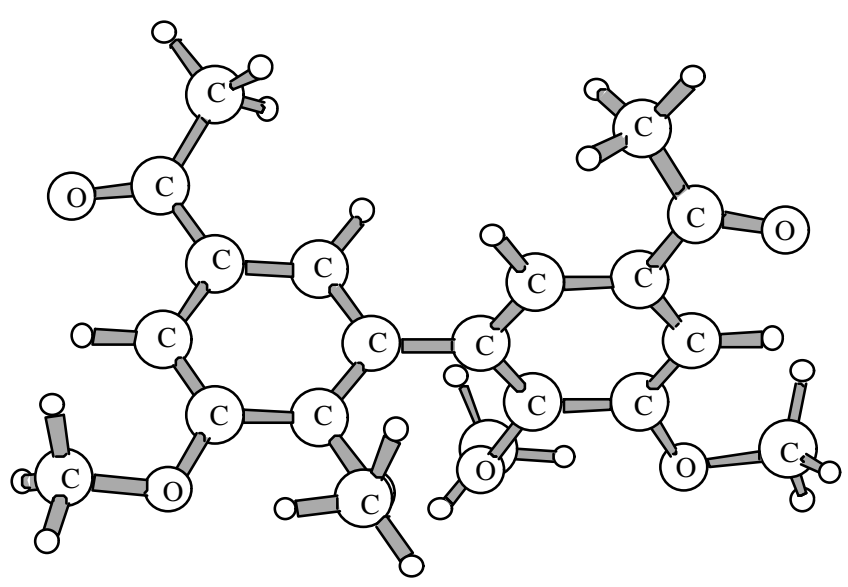

Figura 8. Conformação syn ( $c_{14}$, Tabela 6) obtida em DMSO utilizando a estrutura cristalográfica da molécula DAM como ponto de partida.

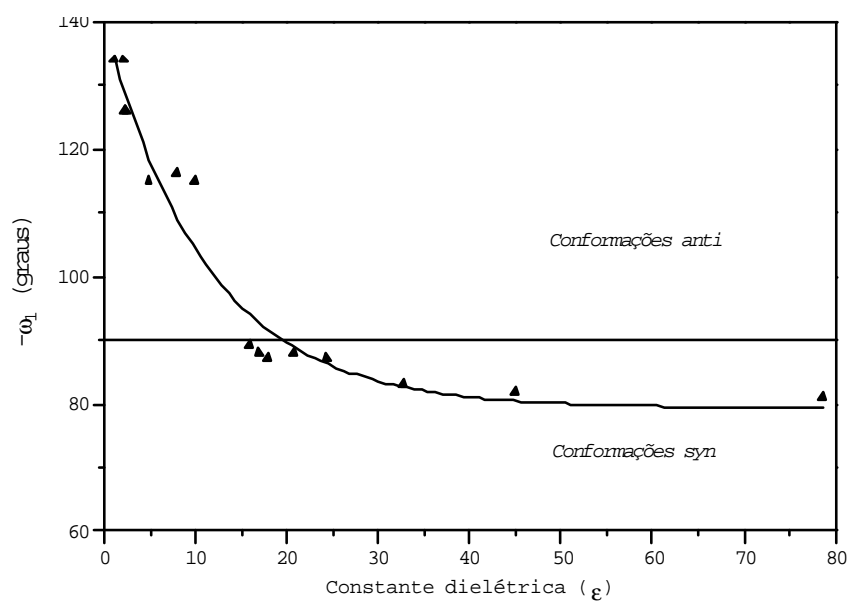

Figura 9. Variação do ângulo torcional interanéis $\left(w_{1}\right)$ como função da constante dielétrica $\varepsilon$ para a molécula DAM. Os valores de $w_{1}$ são referentes às geometrias otimizadas partindo-se da estrutura cristalográfica.

Uma análise similar foi desenvolvida para o derivado DAE. Para este sistema apenas os solventes $\mathrm{CHCl}_{3}$, etanol e DMSO foram considerados, em função da solubilidade $\left(\mathrm{CHCl}_{3}\right.$ e DMSO) e do procedimento de recristalização (etanol). A estrutura anti otimizada em etanol apresentou energia $0,42 \mathrm{kcal} /$ mol acima da estrutura a, enquanto a conformação syn obtida em etanol foi apenas $0,22 \mathrm{kcal} / \mathrm{mol}$ mais estável que a forma $\mathrm{s}_{5}$. Portanto, tem-se que em etanol, a conformação syn obtida nesta última análise (denominada cs-EtOH) e a forma anti $\left(\mathrm{a}_{9}\right)$ correspondem às estruturas de menor energia dentro de cada um dos domínios topológicos. Nesta situação, $\Delta \mathrm{G}_{\mathrm{ij}}^{\mathrm{sol}}$ (anti-syn) foi igual a $-0,11 \mathrm{kcal} / \mathrm{mol}$, o que equivale a uma distribuição de população de $55 \%$ (anti, a a) e $45 \%$ (syn, cs-EtOH). Em $\mathrm{CHCl}_{3}$, as estruturas obtidas apresentaram energias superiores as conformações $a_{2}$ e $s_{2}$. Em DMSO, o equilíbrio é ligeiramente deslocado para a forma syn, sendo $\Delta \mathrm{G}_{\mathrm{ij}}^{\text {sol }}$ (anti-syn) $=0.02$ $\mathrm{kcal} / \mathrm{mol}$, onde a conformação a $\mathrm{a}_{9}$ e a estrutura syn obtida (csDMSO) utilizando a geometria cristalográfica como ponto de partida foram consideradas. Portanto, os resultados desta última análise, relativo a molécula DAE, mostraram que a conformação anti presente em solução corresponde às estruturas $\mathrm{a}_{2}$ $\left(\mathrm{CHCl}_{3}\right)$ e a (etanol e DMSO). A conformação syn, obtida utilizando a geometria cristalográfica como ponto de partida no procedimento de minimização de energia (cs-EtOH e csDMSO), apresentou energia ligeiramente inferior a conformação $s_{5}$ em etanol e DMSO. A Figura 10 representa a estrutura otimizada em DMSO da forma syn cs-DMSO obtida para a molécula DAE.

Finalizando, os resultados obtidos da análise conformacional em solução levaram a seguintes conclusões:

1. A molécula DAM encontra-se na forma syn em solução, sendo a conformação apresentada na Figura $8\left(\mathrm{c}_{14}\right)$ a mais provável em DMSO. Em etanol a conformação $c_{12}$ (Tabela 6) corresponde ao mínimo global na SEP. Em $\mathrm{CHCl}_{3}$ a molécula DAM encontra-se na forma $s_{3}$ (Figura 7a).

2. Para a molécula DAE, a conformação $\mathrm{a}_{2}$ foi obtida como mínimo global em $\mathrm{CHCl}_{3}$. Em etanol um equilíbrio entre $\mathrm{a}_{9}$ e a estrutura syn cs-EtOH foi observado, sendo a concentração relativa igual a: 55\% (anti) e 45\% (syn). Em DMSO, o equilíbrio é ligeiramente deslocado para a forma syn (cs-DMSO) sendo $\Delta \mathrm{G}_{\mathrm{ij}}^{\mathrm{sol}}($ anti-syn $)=0.02 \mathrm{kcal} / \mathrm{mol}$.

Estes resultados justificam a presença de uma única conformação syn para a molécula DAM e duas conformações (anti e syn) para o composto DAE no estado sólido, sendo as estruturas correspondentes àquelas observadas em etanol $\left(\mathrm{c}_{12}\right.$, DAM e cs-EtOH, DAE). As diferenças entre as estruturas teóricas e cristalográficas são atribuídas às interações intermoleculares 


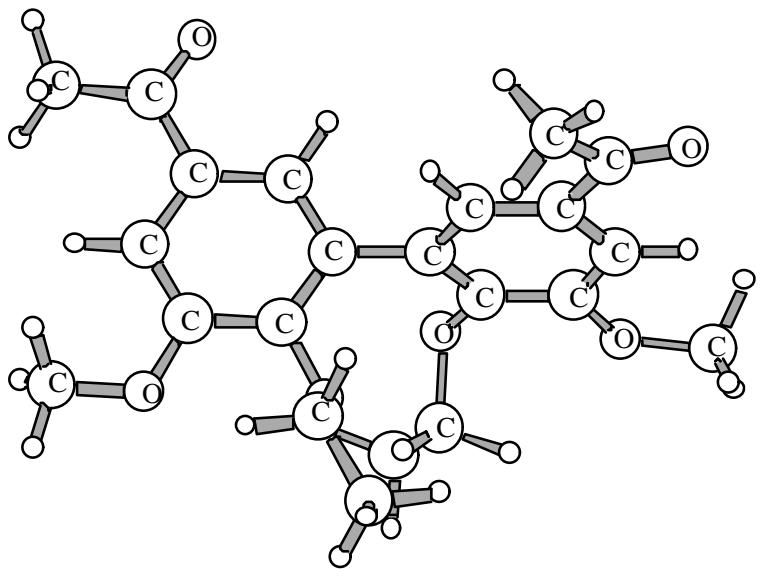

Figura 10. Conformação syn (cs-DMSO) obtida em DMSO utilizando a estrutura cristalográfica da molécula DAE como ponto de partida. Ângulos diedros (em graus): $w_{1}=-83(60), w_{2}=109(-111), w_{3}=-$ $115(128), w_{4}=174(-179), w_{5}=8(-11), w_{6}=166(177), w_{7}=-11(15), w_{8}=-$ $180(-154), w_{9}=179(-151)$. Os valores entre parênteses correspondem aos ângulos torcionais obtidos da estrutura no estado sólido.

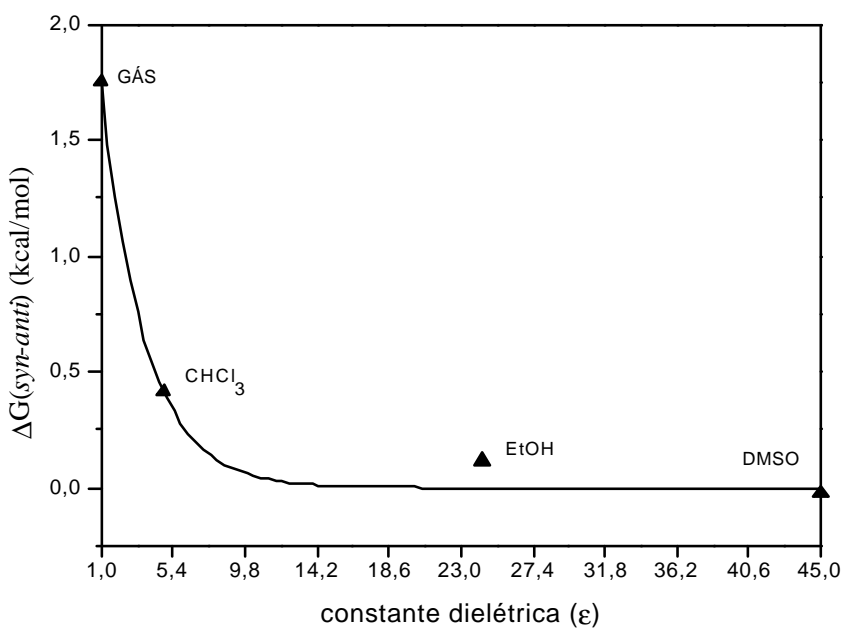

Figura 11. Variação da energia livre de Gibbs ( $\Delta G$ (syn-anti)) em função da constante dielétrica $(\boldsymbol{\varepsilon})$ para a molécula DAE.

presentes na rede cristalina. Os resultados obtidos para o derivado DAM estão também em acordo com a análise de RMN, a qual sugere a presença de uma única forma simétrica presente em $\mathrm{CHCl}_{3}$ e DMSO. Entretanto, para o composto DAE, um equilíbrio entre as conformações syn e anti foi observado em DMSO com uma pequena predominância da conformação syn (cs-DMSO). Em $\mathrm{CHCl}_{3}$ a estrutura anti $\mathrm{a}_{2}$ foi obtida em maior proporção. Os estudos de RMN ${ }^{1} \mathrm{H}$ e ${ }^{13} \mathrm{C}$ apresentaram sinais característicos de uma única forma presente no meio, podendo ser justificado considerando três hipóteses: (i) os sinais no RMN não são sensíveis às mudanças conformacionais, (ii) o processo de interconversão conformacional ocorre em tempo inferior a constante de tempo da técnica e (iii) a conformação syn estaria presente em DMSO e a anti em $\mathrm{CHCl}_{3}$.

A última hipótese mencionada anteriormente pode ser analisada através da Figura 11, a qual mostra o comportamento assintótico da diferença de energia livre de Gibbs entre as conformações anti e syn com o aumento da constante dielétrica. Como observado para os efeitos do solvente na estrutura da molécula DAM, o comportamento apresentado na Figura 11 é conseqüência das aproximações utilizadas no modelo contínuo $\mathrm{COSMO}^{23}$ (eq. 3). Portanto, considerando a tendência de maior estabilização da forma syn em meios polares, pode ser dito que a estabilidade da conformação syn em etanol e DMSO estão subestimadas em relação aos valores obtidos na fase gasosa e em $\mathrm{CHCl}_{3}$, cujos valores de $\varepsilon$ encontram-se em regiões sensíveis do modelo. Esta análise pode também ser utilizada para justificar a baixa concentração da conformação syn em etanol.

\section{CONCLUSÕES}

No presente estudo, uma investigação sistemática da superfície de energia potencial (SEP) para os compostos 4,4'-Odimetildesidrodiacetovanilona (DAM) e 4,4'-O-dietildesidrodiacetovanilona (DAE) foi desenvolvida utilizando a aproximação semi-empírica AM1. Os resultados revelaram diferentes estruturas localizadas em mínimos de energia na SEP. Todas as conformações foram classificadas e agrupadas em domínios topológicos (syn e anti) de acordo com a posição relativa dos grupos $\mathrm{OCH}_{3}$ ligados às posições orto dos anéis aromáticos. Os valores de energia obtidos na fase gasosa, mostraram que as formas anti (moléculas DAM e DAE) correspondem aos mínimos globais na SEP, sendo a diferença de energia livre de Gibbs $<4 \mathrm{kcal} / \mathrm{mol}$ para ambas as espécies.

Em solução, o composto DAM encontra-se predominantemente na forma syn $\left(79 \%\left(\mathrm{CHCl}_{3}\right), 82 \%\right.$ (etanol) e $82 \%$ (DMSO)), em acordo com os estudos de $\mathrm{RMN}^{3}$ e a estrutura observada no estado sólido9. Para a molécula DAE, uma conformação anti foi observada em $\mathrm{CHCl}_{3}(67 \%)$, o que pode ser correlacionado com os sinais de RMN ${ }^{1} \mathrm{H}$ observados ${ }^{3}$. Em etanol, um equilíbrio entre as formas anti $(55 \%)$ e syn $(45 \%)$ foi obtido, justificando as evidências estruturais observadas no estado sólido (os cristais da molécula DAE foram obtidos através de recristalização em etanol). Resultados similares, relacionados ao equilíbrio anti $\rightarrow$ syn, foram obtidos em DMSO, os quais, em princípio, não correspondem as observações experimentais $\left(\mathrm{RMN}^{13} \mathrm{C}\right.$ em $\left.\mathrm{DMSO}^{3}\right)$. Uma possível justificativa foi apresentada considerando os aspectos fundamentais do modelo utilizado na inclusão do efeito do solvente. Portanto, em DMSO, a conformação syn deve corresponder à estrutura mais provável para a molécula DAE.

\section{AGRADECIMENTOS}

O autor gostaria de agradecer as agências de fomento a pesquisa FAPEMIG (Fundação de Amparo a Pesquisa do Estado de Minas Gerais) e CNPq (Centro Nacional de Desenvolvimento Científico e Tecnológico) pelo financiamento parcial do presente projeto e ao CENAPAD-MG/CO-NAR/UFJF (Centro Nacional de Processamento de Alto Desempenho do Estado de Minas Gerais e Região Centro-Oeste) pelos recursos computacionais disponíveis. O autor também agradece ao Prof. W. B. De Almeida (LQC-MM/DQ-ICEx-UFMG) pelas valiosas discussões e sugestões feitas durante o desenvolvimento do trabalho.

\section{REFERÊNCIAS}

1. Sjöstrom, E; Wood Chemistry: Fundamentals and Applications; Academic Press, Inc.; NY, USA, 1981; p 71.

2. Glasser, W. G.; Sarkanen S.; Lignin: Properties and Materials, ACS, NY, USA, 1989; p 2; p 262.

3. Fereira, M. A.; Tese de Doutorado, Depto. Química, UFMG, 1995.

4. Morais, S. A. L.; Tese de Doutorado, Depto. Química, UFMG, 1992.

5. Garver, T. M.; Maa, K. J.; Morat, K.; Can. J. Chem. 1996, 74, 173.

6. Remko, M.; Polein, J.; Z. Phys. Chem. 1980, 120, 1.

7. Shevchenko, S. M.; Barley, G. W.; J. Mol. Struct. (THEOCHEM) 1996, 364, 197.

8. Russel, M. R.; Lim , C.; J. Compt. Chem. 1995, 16, 1181. 9. Ferreira, M. A.; Costa, M. D. D.; Mendes, I. M. C.; 
Drumond, M. G.; P.-Veloso, D.; Fernandes, N. G.; Acta Cryst. 1998, C54, 837.

10. Beusen, D. D.; Berkley-Shands, E. F.; Karasek, S. F.; Marshall, G. R.; Dammkoehler, R. A.; J. Mol. Struct. (THEOCHEM) 1996, 370, 157.

11. Ferguson, D. M.; Raber, D. J.; J. Am. Chem. Soc. 1989, $111,4371$.

12. Saunders, M.; Houk, K. N.; Wu, Yun-Dong; Still, W. C.; Lipton, M.; Chang, G.; Guida, W. C.; J. Am. Chem. Soc. 1990, 112, 1419.

13. Rocha, W. R.; Pliego, Jr, J. R.; Resende, S. M.; Dos Santos, H. F.; De Oliveira, M. A.; De Almeida, W. B.; $J$. Comput. Chem. 1998, 19, 524.

14. Dos Santos, H. F.; Rocha, W. R.; De Almeida, W. B.; $3^{o}$ Congresso Brasileiro de Polímeros 1995, 1, 1199.

15. De Almeida, W. B.; Dos Santos, H. F.; O’Malley, P. J.; Struct. Chem. 1995, 6, 383.

16. Dos Santos, H. F.; De Almeida, W. B.; Taylor-Gomes, J.; Booth, B. L.; Vib. Spectrosc. 1995, 10, 13.

17. Silva, T. H. A.; Oliveira, A. B.; De Almeida, W. B.; Struct. Chem. 1997, 8, 95.

18. Silva, T. H. A.; Oliveira, A. B.; De Almeida, W. B.; Biorg. Med. Chem. 1997, 5, 353.

19. Mezey, P. G.: Studies in Physical and Theoretical Chemistry Potential Energy Hypersurfaces, Elsevier, 1987.

20. Dewar, M. J. S.; Zoebish, E. G.; Healy, E. F.; Stewart, J.
J. P.; J. Am. Chem. Soc. 1985, 107, 3902.

21. Mopac 93.00 Manual, Stewart, J. J. P., Fujitsu Limited, Tokyo, Japan (1993).

22. Baker, J.; J. Compt. Chem. 1996, 7, 385.

23. Klant, A.; Schüürmann, G.; J. Chem. Soc. Perkin Trans. 2 1993, 799.

24. Bastiansen, O.; Acta Chem. Scand. 1949, 3, 408.

25. (a) Trotter, J.; Acta Cryst. 1961, 14, 1135; (b) Hargreaves, A.; Rizvi, S. H.; Acta Cryst. 1962, 15, 365.

26. Eliel, E. L.: Stereochemistry of Carbon Compounds, McGraw-Hill Book Company, Inc., BY, USA, 1962; p 124.

27. Kistiakowsky, G. B.; Smith, W. R.; J. Am. Chem. Soc. 1936, 58, 1043.

28. O'Shaughnessy, M. T.; Rodebush, W. H.; J. Am. Chem. Soc. 1940, 62, 2906.

29. Westheimer, F. H.; Mayer, J. E.; J. Chem. Phys. 1946, $14,733$.

30. Desponds, O.; Schlosser, M.; Tetrahedron Lett. 1996, 37, 47.

31. Do Val, A. M. G.; Guimarães, A. C.; e De Almeida, W. B.; J. Heterocyc. Chem. 1995, 32, 557.

32. Alcântara, A. F. de C.; P.-Veloso, D.; Stumpf, H. O.; De Almeida, W. B.; Tetrahedron 1997, 53, 16911.

33. Klaeboe, P.; Vib. Spectrosc. 1995, 9, 3.

34. Lide, D. R. Ed.: Handbook of Chemistry and Physics, $73^{\mathrm{RD}}$ Edition, CRC Press, London, UK, 1992-1993. 